\title{
On a Non-linear Risk Analysis for Stock Market Indexes
}

\author{
Kenjiro Suzuki · Yasunori Okabe • Takaaki Fujii
}

Published online: 10 August 2007

CSpringer Science+Business Media, LLC 2007

\section{Erratum to: Asia-Pacific Finan Markets DOI: $10.1007 / \mathrm{s} 10690-007-9043-z$}

Please note that the author affiliations in the original article are incorrect. The correct affiliation per author should be as shown below.

The online version of the original article can be found under doi:10.1007/s10690-007-9043-z.

K. Suzuki $(\varangle)$

Mitsubishi Research Institute Inc. 3-6, Otemachi 2-Chome, 100-8141 Chiyoda-ku, Tokyo, Japan e-mail: ksuzuki@mri.co.jp

Y. Okabe

Department of Mathematics, School of Science and Technology, Meiji University, 1-1-1 Higashimita, Tama-ku, Kawasakishi 214-8571, Japan

e-mail: okabe@math.meiji.ac.jp

T. Fujii

Aioi Insurance Co. Ltd. 1-28-1, Ebisu, Shibuya-ku, Tokyo 150-8488, Japan

e-mail: Takaaki-fujii@ioi-sonpo.co.jp 